(C) 2012

SAMUEL GHEBREMARIAM

ALL RIGHTS RESERVED 


\title{
ENERGY PRODUCTION COST AND PAR MINIMIZATION IN MULTI-SOURCE POWER NETWORKS
}

\author{
A Thesis \\ Presented to \\ The Graduate Faculty of The University of Akron \\ In Partial Fulfillment \\ of the Requirements for the Degree \\ Master of Science
}

Samuel Ghebremariam

May, 2012 


\title{
ENERGY PRODUCTION COST AND PAR MINIMIZATION IN MULTI-SOURCE POWER NETWORKS
}

\author{
Samuel Ghebremariam
}

Thesis

Approved:

Accepted:

Advisor

Dr. Hamid Reza Bahrami

Dean of the College

Dr. George K. Haritos

Faculty Reader

Dean of the Graduate School

Dr. Yilmaz Sozer

Dr. George R. Newkome

Faculty Reader

Dr. Malik Elbuluk

Date

Department Chair

Dr. Alex De Abreu Garcia 


\section{ABSTRACT}

There is a growing demand for the electricity in the world. This fact and the need for modernization of power generation and distribution networks, calls for novel ways to generate, manage, and distribute the electricity. Smart grid is believed to be a viable technology to make the power networks more efficient by managing the energy sources and the consumer loads throughout the newtrok. This management can be done at the utility side or the load side and it requires an autonomous network with two-way communication among the loads and the sources that provides extensive real-time information exchange between the generation nodes, consumer loads and all the switching points in between.

The aim of this thesis is to study the problem of minimizing the power generation cost and peak-to-average ratio (PAR) of sources' energy production for a network of multiple energy sources. We consider a power distribution network in which each load is connected to multiple sources and depending on network connectivity can be fed by more than one power source. It is assumed that the power generation cost changes from one power source to another and that the power generation cost for each source is a function of its load. Based on the network model and assuming the power consumption schedules for all the loads are available, we then introduce two different 
optimization problems to minimize the overall cost of energy production throughout the network and to minimize the PAR of sources' energy. Further, we introduce a hybrid optimization problem that combines the energy production cost and PAR objective functions with a proper weight. By solving these optimization problems using convex optimization techniques, we find out the best load sharing schemes that minimize the derived objective functions. In practice, each load shares its consumption schedule hourly (or daily) with the central power network administrator over a wired or wireless area network. This information can then be used to solve the formulated optimization problems. Simulation results confirm that the resulting load sharing schemes bring about significant reduction in the energy production cost as well as the PAR of the required generated energy throughout the network. 


\section{ACKNOWLEDGEMENTS}

First and foremost, I would like to thank God for helping me achieve success in my studies.

This thesis would not have been possible without the support of many people. I would like to express my gratitude to my advisor, Dr. Hamid Reza Bahrami who was abundantly helpful and offered invaluable assistance, support and guidance. Deepest gratitude is also due to the members of my supervisory committee, Dr. Yilmaz Sozer and Dr. Malik Elbuluk without whose knowledge and assistance this study would not have been successful.

Special thanks also to all my graduate friends, especially Wireless Communication Lab partners; Sajjad Beygi, Mehdi Sadeghzadeh, Ahmad Danaee, Krishna Budhatoki, Mohammed El-tayeb, Shashwat Jnawali, Mehdi Maleki and Mohammed Kafashan for their contribution to my thesis and invaluable assistance. I would also like to thank my best friends in Akron, Mina Shahata, Anna Herr, Joe Novak, Lucas Gagne and Poest's who always been there in my time of need.

I would also like to convey thanks to the faculties in Department of Electrical and Computer Engineering and head of the department Dr. Alex De Abreu Garcia for giving me this wonderful opportunity to pursue my studies and reach this point. 
Last but not least, I would like to express my love and gratitude to my beloved families in Asmara and Indianapolis for their understanding and endless love through the duration of my studies. 


\section{TABLE OF CONTENTS}

\section{Page}

LIST OF TABLES ........................... . . ix

LIST OF FIGURES . . . . . . . . . . . . . . . . . . .

\section{CHAPTER}

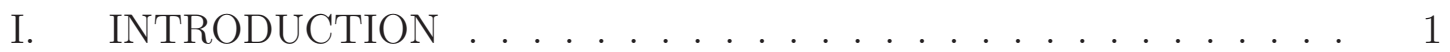

1.1 Existing Electric Grid . . . . . . . . . . . . . . . . . 1

1.2 Defining Smart Grid . . . . . . . . . . . . . . . . . . . . 2

1.3 Motivation for Smart Grid . . . . . . . . . . . . . . . . . 4

1.4 Components of Smart Grid . . . . . . . . . . . . . . . . . 5

1.5 Problem Statement and Previous Work in Literature . . . . . . . . 6

1.6 Overview and Contribution of the Thesis . . . . . . . . . 8

II. ENERGY MANAGEMENT . . . . . . . . . . . . . . . . . . . . . . . 12

2.1 Load Management . . . . . . . . . . . . . . . . . . . . . 12

2.2 Demand Response . . . . . . . . . . . . . . . . . . 18

2.3 Demand Management versus Demand Response . . . . . . . . . . 19

2.4 Nature of Loads . . . . . . . . . . . . . . . . . . . . . . . . . . . . 20 
2.5 Supply Side Management . . . . . . . . . . . . . . . . . 21

2.6 Economic Dispatch . . . . . . . . . . . . . . . . 22

III. MULTI-SOURCE POWER NETWORK WITH FIXED LOADS . . 24

3.1 System Model . . . . . . . . . . . . . . . . . . . . 24

3.2 Problem Setup and Solution . . . . . . . . . . . . . . . 27

3.3 Simulation Results . . . . . . . . . . . . . . . . . . . . . . 31

IV. MULTI-SOURCE POWER NETWORK WITH SHIFTABLE LOADS 36

4.1 System Model . . . . . . . . . . . . . . . . . 36

4.2 Problem Setup . . . . . . . . . . . . . . . . . . . . . . . . 39

4.3 Simulation Results . . . . . . . . . . . . . . . . . . . 45

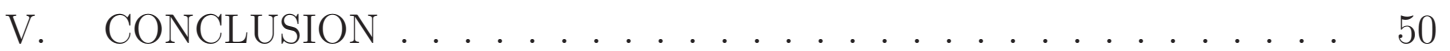

BIBLIOGRAPHY . . . . . . . . . . . . . . . . . . . . 52 


\section{LIST OF TABLES}

Table

Page

1.1 Comparison of Existing Grid and Smart Grid . . . . . . . . . . . . . 3

1.2 Communication Technologies for Smart Grid [1] . . . . . . . . . . . . 11

4.1 System Parameters . . . . . . . . . . . . . . . . . 40

4.2 Proposed Optimization Algorithm . . . . . . . . . . . . . 43 


\section{LIST OF FIGURES}

Figure

Page

1.1 Components of a smart grid $[12] \ldots \ldots \ldots \ldots$

2.1 A smart energy profile $[38] \ldots \ldots \ldots \ldots$. . . . . . . . . . . 14

2.2 Examples of two non-flat pricing schemes $[30] \ldots \ldots$

3.1 A simple interconnected multi-source power network . . . . . . . . . 26

3.2 Normalized retail total energy cost for $N=4$ users and $M=3$ sources; Top: random load sharing, Center: hybrid load sharing, Bottom: minimum cost load sharing . . . . . . . . . . . . . . . . . . 33

3.3 PAR for the random and minimum cost load sharing schemes . . . . . . 34

3.4 PAR for minimum cost, minimum PAR and hyrbid load sharing schemes 35

4.1 Load distribution before optimization . . . . . . . . . . . . . . . 47

4.2 Load distribution after optimization over the interval of consumption . 48

4.3 Daily charges for each load before and after optimization . . . . . . . 48

4.4 PAR performance: without optimization, with cost minimization, and with PAR minimization schemes . . . . . . . . . . . . . . . . 49 


\section{CHAPTER I \\ INTRODUCTION}

\subsection{Existing Electric Grid}

There are currently 9200 electricity generating units with 300,000 miles of transmission and distribution lines in the United States. The National Academy of Engineering refers to the existing grid system as the greatest engineering achievement of the 20th century due to its reliability [1]. However, today's grid is aging, inefficient, and inadequate for the increasing electricity demand. It is also outdated in many aspects: it cannot integrate new (alternative) energy resources efficiently; and it cannot accommodate properly new consumer loads such as plug-in hybrid electric vehicles. The main drawbacks of the existing grid system are:

i) Reliability: The increasing demand for energy can cause more blackouts. This happens due to slow mechanical switches, lack of automated analysis and lack of situational awareness. It is estimated that the growth in peak demand exceeds growth in generation by almost $25 \%$ every year and this results in power outage cost of 180 billion per year [2].

ii) Energy Efficiency: The efficiency of the current power generation and distribution network is low. Higher energy efficiency can be translated into lower emission 
and electricity cost. Studies show that if the current grid were just $5 \%$ more efficient, the energy savings would equate to eliminating 53 million cars [3].

iii) Affordability: The rising fossil fuel cost triggers an increase in energy cost which in turn makes electricity unaffordable to consumers [4]. It is possible to reduce the cost of electricity by incorporating renewable and distributed energy sources into the grid. However, as explained, it is very difficult, if not impossible, to integrate such energy sources in the current grid.

\subsection{Defining Smart Grid}

"Smart Grid is a necessary response to the environmental, social, and political demands, placed on energy supply." [5]

It would be quite difficult to draw a clear defining line between "smart grid" and the current grid. It is more practical to consider "smart grid" as an improvement to the existing power system. It is a digitally enabled grid with an intelligent communication infrastructure. This two-way communication among loads and utilities enables timely, secure, and adaptable information flow that is needed to improve the reliability and energy efficiently [6].

Smart Grid is best known for the following features [7]:

- Optimized resource and equipment utilization

- Distributed by its structure

- Interactive, adaptive and scalable 
Table 1.1: Comparison of Existing Grid and Smart Grid

\begin{tabular}{|l|l|}
\hline Existing Grid & Smart Grids \\
\hline Centralized power generation & Distributed power generation \\
\hline One-way power flow & Two-way power flow \\
\hline Less reliable & Way reliable than the existing \\
\hline Limited grid accessibility for new sources & Loads follow generation \\
\hline Limited Control & Pervasive control \\
\hline Few customer choices & Many customer choices \\
\hline Failure and blackouts & Adaptive and islanding \\
\hline Manual restoration & Self-healing \\
\hline
\end{tabular}

- Proactive rather than reactive

- Self-healing and environment friendly

- Reliable, secure and efficient

- Open for all types and sizes of generation

Table 1.1 provides a comparison between the existing (traditional) grid and smart grid [7]-[8]. 


\subsection{Motivation for Smart Grid}

The main driving force for the introduction of smart grid is the advancement of the current technology. We now have new energy production, conversion, and storage technologies that may reduce the need for additional power plants in the network; and can cut the cost of generation and/or failure [9]. The other driving factor of smart grid is the scarcity of energy sources. This gives rise to energy source optimization (e.g. distributed generation and energy storage) as well as optimization of time of use (e.g. avoiding peak hours usage). It is also possible to introduce efficient pricing schemes that alter the consumers' behavior. The pricing schemes that are going to be used for the future grid system aim at reducing demand during the peak usage periods [7]. To motivate customers lower their energy use, electricity price is increased during high demand periods. These variable pricing schemes are used in this thesis to formulate optimization problems to reduce the energy generation cost and other network parameters. This will be discussed further in the next chapters.

Besides the above points, the power quality, safety, integrating renewable energy sources, peak levelling and time of use pricing are some important features that the current grid system has failed to provide efficiently [10]. This further motivates the introduction of smart grid. 


\subsection{Components of Smart Grid}

A smart grid is a widely distributed electricity delivery network characterized by twoway flow of electricity and information. It is capable of monitoring every component in the network from power plants to customer appliances. A smart grid is comprised of different parts that help the system reach its intended performance. The following list includes the key components of a smart grid [11]:

- Integrated communication

- Sensing and measurement

- Advanced components

- Advanced control methods

- Improved interfaces and decisions support

Figure 1.1 shows basic building blocks of a smart grid.

For instance, the communication network is an important part of a smart grid. It enables the grid to become a dynamic and interactive medium for two-way real time flow of information and power[13]. It further enhances reliability through integrating Demand Response (DR) and also enhances security through detection of physical and cyber treats to the grid. Table 1.2 lists the possible communication technologies that could be used in a smart grid. 


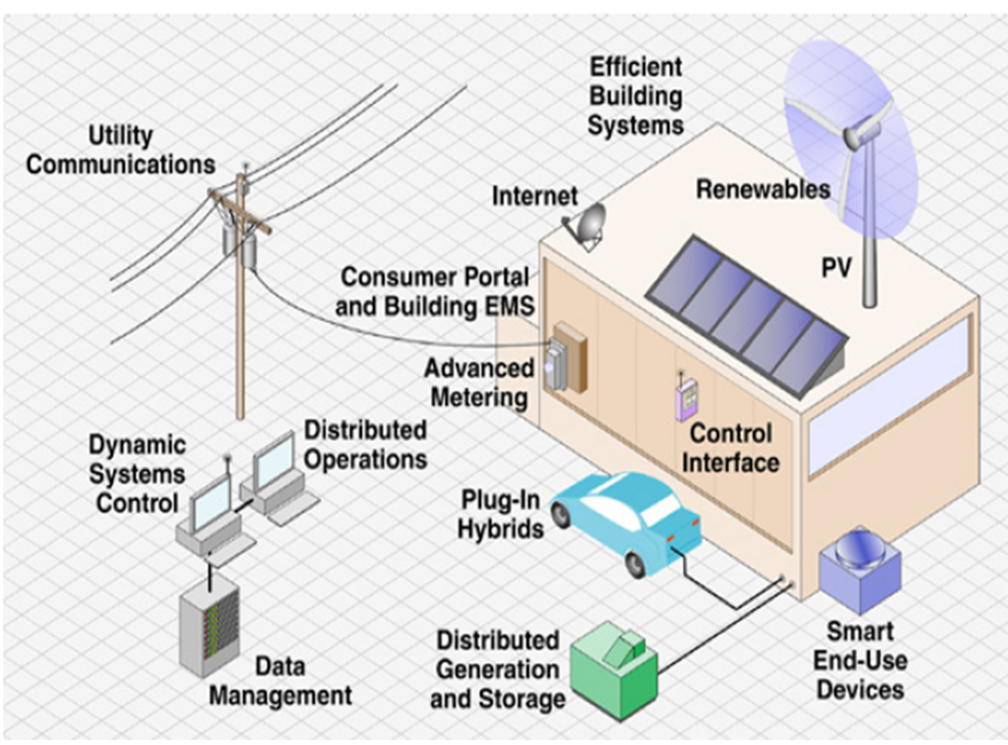

(from the GridWise Alliance's Realizing the Value of an Optimized Electric Grid)

Figure 1.1: Components of a smart grid [12]

\subsection{Problem Statement and Previous Work in Literature}

Electrical energy is a form of energy that is difficult to store in bulk. After generation, it must be taken to the point where it needs to be used. Consequently, in the generation and distribution of electrical energy, source-load management is an issue that continuously concerns the power network operators. Failure in providing a sourceload balance in the network will cause system instability and blackouts. In case that the overall system load approaches or exceeds the maximum generating capacity of the network, the network operator should either find additional supplies of energy that requires introduction of distributed energy resources (DER) or find ways to limit the increasing load demand. The former option requires small-scale power generation 
technologies typically in the range of $3 \mathrm{~kW}$ to $10,000 \mathrm{~kW}$ to provide an alternative or an enhancement to the traditional electric power system. Limiting incoming demand seems partly feasible by introducing policies such as variable pricing schemes that encourage lower consumption while penalizing excessive energy usage.

On the other hand, due to the rapid increase in the electrical energy consumption, the average retail price of electricity to the end consumers has been constantly increasing in the past few years. Maintaining a proper source-load balance throughout the network, utilizing the limited network resources more efficiently and introducing variable pricing schemes are key elements to improve the efficiency of power networks and to control the cost of energy generation and distribution.

Several studies have been conducted on the Demand Side Management (DSM) focusing primarily on the interactions between a utility company and consumers [14]-[17]. A variety of DSM schemes have been introduced to efficiently manage the use of energy in the network and to supervise residential and commercial loads [14]-[16]. A software system is developed to directly control the residential loads through communicating with the loads [17]. This is sometimes referred to as Direct Load control (DLC). DLC is an approach for residential load management in which the utility company can remotely control the operations of certain appliances in a household. Several DLC algorithms have been developed to determine the optimal load control schedules of groups of domestic appliances [18]-[25]. The majority of these schemes are based on linear programming or dynamic programming and try to minimize peak 
load or electricity production cost over a certain time period. Several papers have studied the problem of Economic Dispatch that aims at minimizing the total cost of energy generation by either sharing the generation between generators or shifting generation from more expensive to less expensive sources [26]-[28]. Other studies suggest autonomous and distributed demand side management using two-way communication between consumer loads and the network [29].

Introduction of two-way communication between the loads and the network provides an additional degree of flexibility in optimizing power networks. Using this concept, an optimal residential load control scheme introduced in [30] that focuses on the integration of real time pricing (RTP) and inclining block rates (IBR) to achieve a trade-off between the electricity cost and the waiting time for the operation of each load in the system. [30] also develops optimal consumption schedules to reduce the peak-to-average-ratio (PAR) of energy consumption for the loads in the network.

\subsection{Overview and Contribution of the Thesis}

While the approach in [30] studies the cost and the PAR minimization from the load side, it is also reasonable to consider the same problem from the source side. The approach is more effective to reduce the overall power generation and distribution cost and the sources' generated energy PAR in the network. Reducing the PAR is important because it results in further efficiency, lower required network capacity, lower maintenance cost and longer life span of the power stations in the network [31]. 
In this thesis, schemes to minimize the cost of energy production or the PAR for a multi-source power network are considered. It is assumed that there are multiple sources in the network that are connected to several loads. The consumption schedules of the loads throughout a span of time are communicated to the network. The load schedules are assumed to be fixed or flexible throughout the time span. Several optimization problem to minimize the cost of electricity generation, the PAR or a combination of these two are then formulated and solved using numerical optimization techniques.

There are several work in the literature on Economic Dispatch that aim at minimizing the total cost of generation by either sharing the generation between generators or shifting generation from the more expensive to less expensive generators [32]-[34]. The approach proposed in this thesis is different from the Economic Dispatch problem as it also incorporates the consumption schedule of the loads into the optimization problem and also takes into account the PAR performance of each source unit. It is assumed that each residential load is equipped with a smart meter with two-way digital communication capability connected to a smart power distribution system. The smart meters calculate and send their energy consumption schedule to the central network administrator on an hourly (or daily) basis. We then develop a suitable system model that is used to formulate two constrained optimization problems to find the optimum load sharing scheme among energy sources to minimize the overall cost of electricity generation throughout the network and the PAR of the required 
energy generation. As a third optimization problem, a hybrid case in which the target function is the weighted sum of the cost and PAR minimization problems is considered. This last optimization problem is useful when the focus is on reducing both energy generation cost and the PAR. The derived optimization problems are convex and non-linear programming techniques such as the interior point method can be used to solve them. Through simulation, it is shown that the proposed scheme can significantly reduce the cost and the PAR of electricity generation in a multi-source power generation and distribution network. 
Table 1.2: Communication Technologies for Smart Grid [1]

\begin{tabular}{ll}
\hline Technology & Comments \\
\hline Broadband Over & \\
Power Line & $\bullet$ Communication over low and medium voltage lines \\
& $\bullet$ Currently used for Automatic Meter Reading (AMR), DR, DER
\end{tabular}
Multiple Address
System Radio
- Consists of a master radio transmitter/receiver and several remote transmitters/receivers
- Currently deployed for Supervisory Control and Data Acquisition (SCADA) and Distribution Automation (DA)

Spread Spectrum

Radio Systems

- Point to multi-point, secure radio system where frequency hops in a particular band

- Used as last mile connection to a main communication system

WiFi

- Short range wireless communication based on IEEE $802.11 \mathrm{~b}$ and IEEE 802.11g standards

- Useful for communication within the premises

WiMax

- 4G wireless technology that provides longer distance communications (10-30 miles) with data rates of $75 \mathrm{Mbps}$

- Can be used as the core transmission and distribution communications system that supports WiFi applications for SA or DA 


\section{CHAPTER II}

\section{ENERGY MANAGEMENT}

\subsection{Load Management}

Load (demand side) management is the process of balancing the supply of electricity in the network with the demand by adjusting or controlling the load rather than the electricity generation at the power stations. Load management allows utilities to reduce the demand for electricity during peak usage times which can in turn reduce costs by eliminating the need for peaking power plants.

Load Management was originally considered to include any action on the demand side that changes the shape of a customer's energy load profile [35]. It refers to cooperative

activities between the utility and its customers (sometimes with the assistance of third parties such as energy services companies and various trade allies) to implement options for increasing the efficiency of energy utilization with benefits to the customer, the utility, and the society as a whole [35]. Some of the reasons for load management are as follows [37]:

- Improve the efficiency of energy systems through improved generation efficiency and system load factor

- Reduce financial needs to build new energy facilities (generation) 
- Lower the cost of delivered energy to consumers, lower generation costs and lower customer bills through the use of energy efficient equipment and appliances

- Reduce power shortages and power cuts i.e. improved system reliability though decrease in demand

- Improve the reliability and quality of power supply through demand reduction in distribution systems

Load Management programs include conservation and energy efficiency programs, fuel substitution programs, demand response programs and residential or commercial load management programs [14]. Residential or commercial load management programs usually aim at one or both of the following design objectives: reducing consumption and shifting consumption.

Figure 2.1 shows a smart energy application profile. It promises to be a key element of residential energy (load consumption) management systems. It is capable of passing energy-related messages across any wired or wireless home area network.

There are three possible approaches to perform load management. These include direct load control (DLC), dynamic pricing and load shifting.

\subsubsection{Direct Load Control}

DLC is an approach for residential load management in which the utility company can remotely control the operations of certain appliances in a household [25]. Utilities have different objectives for load control including objectives related to operating cost, 


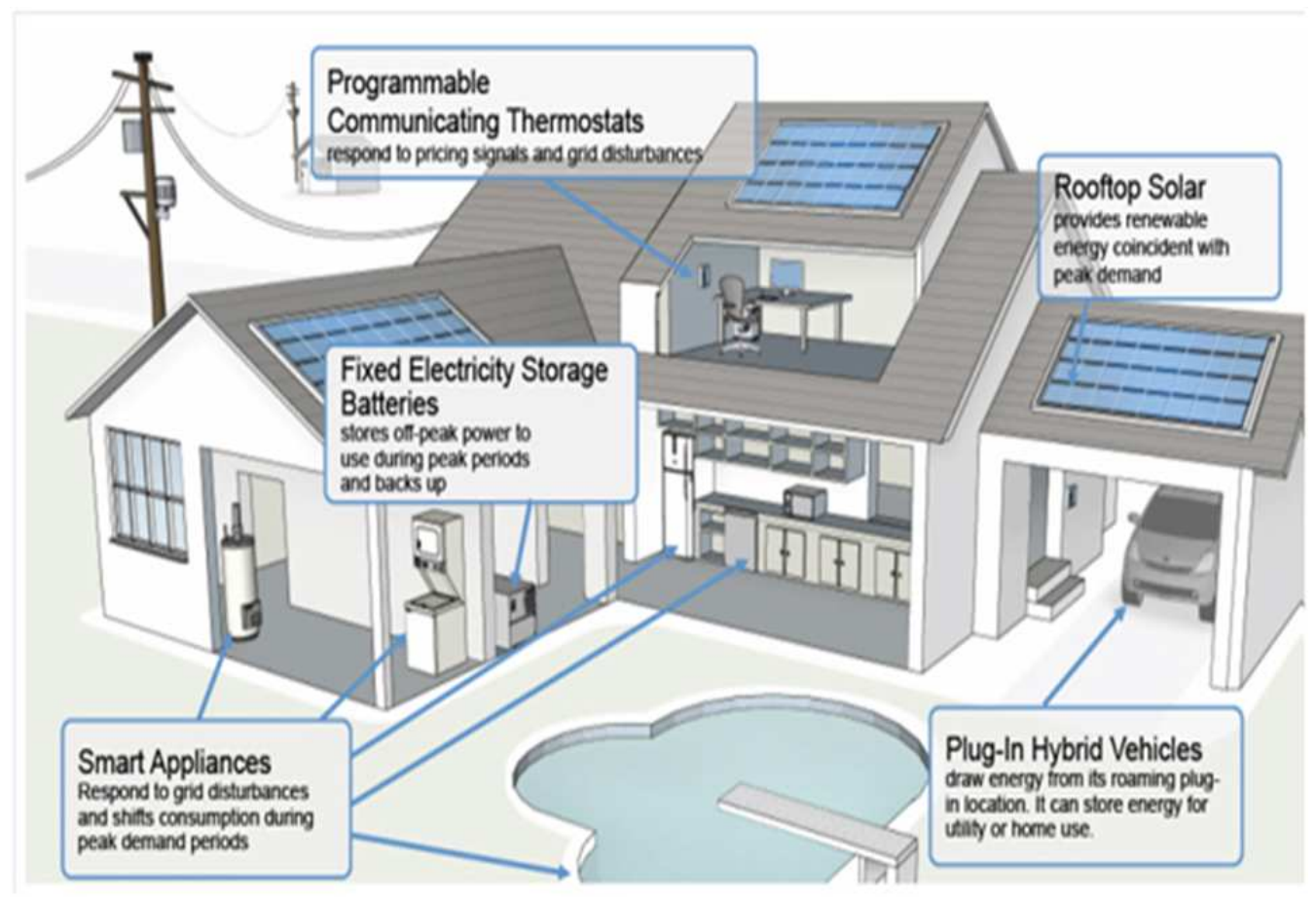

Figure 2.1: A smart energy profile [38] 
system security and load research. Two common objectives are the minimization of the peak load and the production cost.

\subsubsection{Dynamic Pricing}

Dynamic pricing is an electricity pricing available to the customer in regular intervals in which the consumer does not typically know the electricity prices more than a day in advance. Dynamic pricing incentivizes electricity customers to lower their usage during peak times, especially during the critical hours of peak demand [39]. These pricing schemes might be classified as time-varying rate structures or static rate schemes. Some of the time-varying schemes include real-time pricing (RTP), time of use pricing and peak pricing whereas flat and tiered rates (inclined/declined

block rates or IBR) are the non-time varying schemes. Figure 2.2 shows examples of two promising non-flat pricing models, i.e. IBR and RTP.

\section{Flat Rates}

It refers to a pricing structure that charges a single fixed fee for a service regardless of usage. Rarely, it may refer to a rate that does not vary with usage or time of use. Flat rates are typically the same over all hours of usage [40].

\section{Tiered Rates}

Tiered rates vary by the level of energy usage in the billing period which may be fixed blocks (inclining or declining) for the class or customer-specific. In inclining block rate (IBR) pricing, the marginal price increases by the total quantity consumed [41]. 


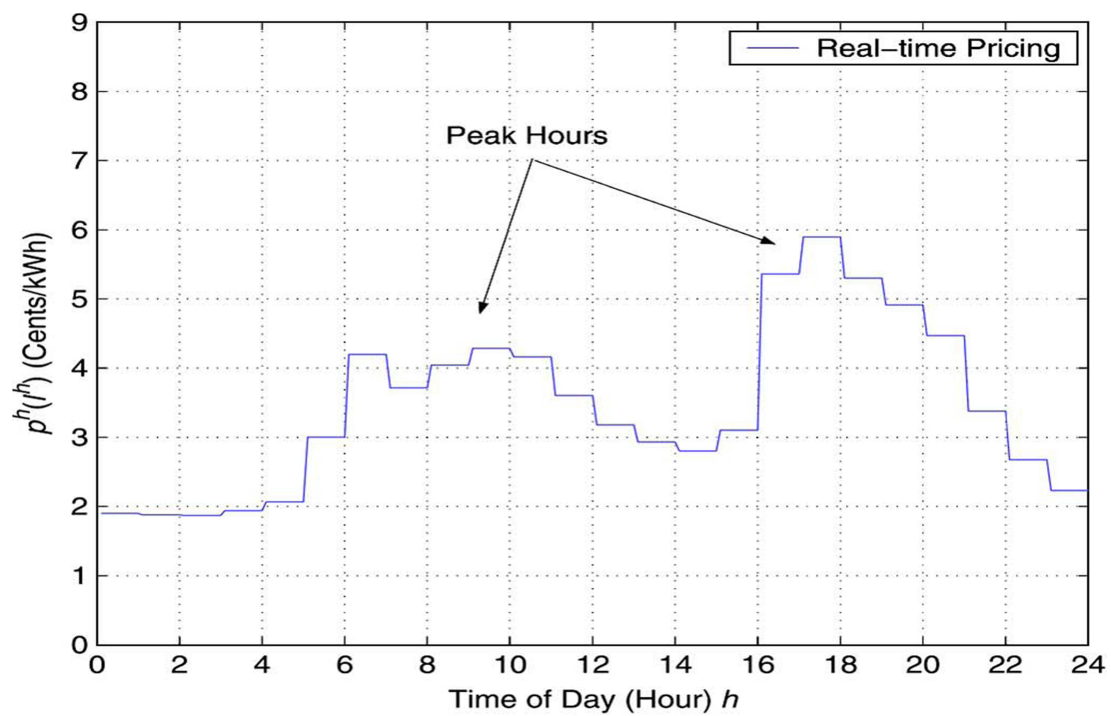

(a)

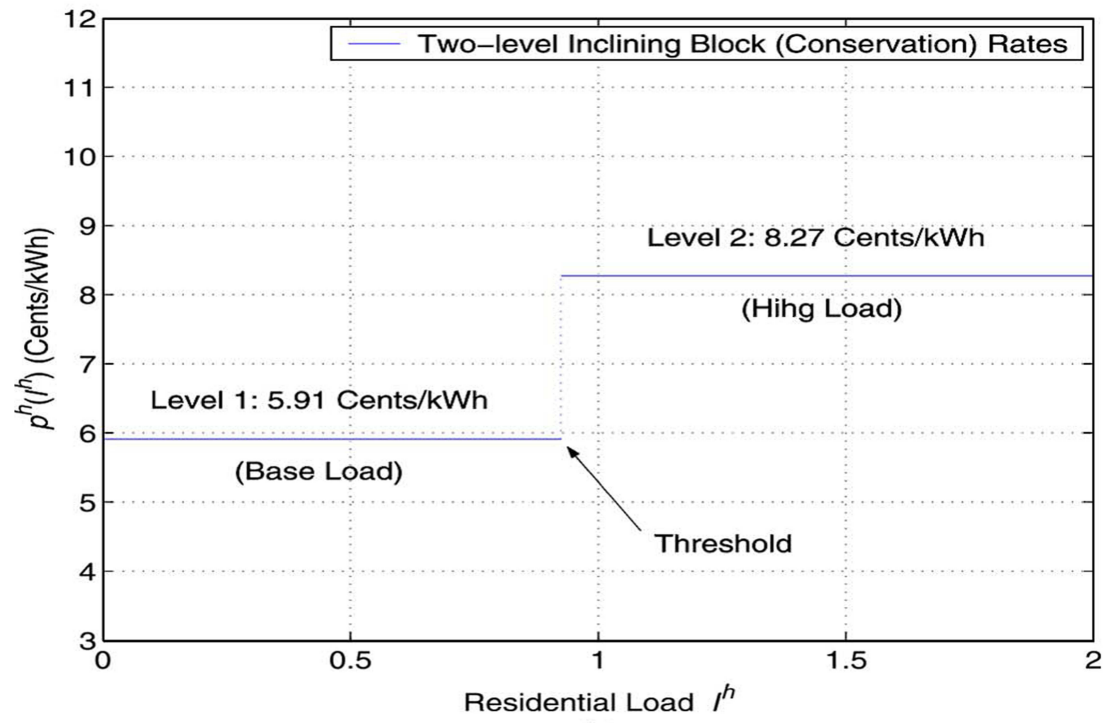

(b)

Figure 2.2: Examples of two non-flat pricing schemes [30] 
That is, beyond a certain threshold in the total monthly/daily/hourly residential load, the electricity price will increase to a higher value. This creates incentives for end users to conserve to distribute their load at different times of the day in order to avoid paying for electricity at higher rates. In addition, IBR may help in load balancing and reducing the PAR [42].

\section{Real-Time Pricing (RTP)}

Electricity prices vary continuously throughout the day as a function of environmental conditions (such as outdoor air temperature) or electricity supply and demand conditions. Real-time prices can be set with day-ahead or day-of schedules. Prices usually vary hourly [43].

\section{Peak Pricing}

In peak pricing, electricity prices on peak days are different than electricity prices on non-peak days. Prices are generally preset; however, the customer does not know if a certain day will be a peak day or a non-peak day until day-ahead or day-of [44]. Peak days are called as a function of environmental conditions (such as weather forecasts) and/or electricity supply and demand conditions.

\section{Time-Of-Use Pricing (ToUP)}

ToUP is not considered a form of dynamic pricing because it follows a fixed schedule. We define ToUP as electricity pricing in which the consumer knows his or her elec- 
tricity prices more than a day in advance, though the price varies throughout the day [45]. In ToUP, the time periods and prices are often fixed at least a year in advance.

\subsubsection{Load Shifting}

It is a load management technique that aims to move demand from the peak hours to off-peak hours of the day i.e. hourly loads are shifted from peak price periods (hours) to non-peak price periods. This accounts for shifting all of the load or some portion of it $[46]$.

\subsection{Demand Response}

Demand response (DR) activities are defined as "actions voluntarily taken by a consumer to adjust the amount or timing of his energy consumption. Actions are generally in response to an economic signal (e.g. energy price, or government and/or utility incentive)" [37]. The three possible types of DR actions are price response, demand bidding and voluntary load shedding.

\subsubsection{Price Response}

Price response refers to situations where customers voluntarily reduce energy demand due to high prices during times of peak demand. In return for shedding some or all of their electric load when wholesale prices are high, utilities will make client payments based on the wholesale electricity price [47]. Essentially, the more kilowatt hours a client can shed, the more money they will make. Utilities notify clients when prices are 
anticipated to exceed the client's pre-determined threshold. Participation is typically voluntary; so a client may choose whether to shed load on a given day or not.

\subsubsection{Demand Bidding}

In demand bidding, large customers could "sell" their reductions in demand to the utilities in times of peak demand. When the energy demand exceeds its local supply, it may be less expensive to pay large users to reduce their demand than to import electricity from different sources [37].

\subsubsection{Voluntary Load Shedding}

Voluntary load shedding (VLS) refers to situations where customers voluntarily reduce energy demand in response to appeals from the government during times of high demand and/or constrained supply. VLS means a customer signs a contract with the supplier to be load shed up to some percentage during specified peak time, and the supplier pays an incentive for the amount of electricity shed and ensures electricity supply for the period of the VLS [48].

\subsection{Demand Management versus Demand Response}

DSM and DR share some similarities in that both are mechanisms to improve the energy efficiency in a power network. They, however, have differences on how well the energy reduction is achieved [37]. DSM is focused on achieving sustained energy use 
reductions and is often driven by incentives whereas DR is market driven and results in temporary reductions or temporal shifts in energy use.

\subsection{Nature of Loads}

Loads within a power system can be very diverse. The classical customer classifications on [49] separates customers into three main groups:

- Domestic customers

- Commercial customers

- Industrial customers

These groups can also be further separated into subgroups formed by customers with similar load consumption patterns. For each of these groups or subgroups daily load consumption curves have been derived. Obviously, these load curves depend on many variables and vary largely from country to country and even from region to region. The expected load consumption of a customer of any group at a certain time can be decomposed into the following parts according to controllability [50]:

- Non controllable part of the load

- Controllable part of the load

- Load that can be shifted

- Load that can be curtailed 
The part that can be shifted is the load that can be moved to some other time periods in order to improve system benefits. This load is not going to disappear; the consumption is just going to be postponed. Examples of this kind of load in the residential sector are:

- Washing machines

- Dryers

- Dishwashers

- Water heaters

The load that can be curtailed is load that can be eliminated. Examples of this kind of load in the residential sector are:

- Air-conditioners

- Space-heaters

\subsection{Supply Side Management}

In contrast to DSM, the supply or grid side management (GSM) are the measures taken by the utilities on power plants and transmission and distribution lines in order to minimize the cost of energy for a given level of reliability, and to reduce the impact on the environment [51]. 


\subsection{Economic Dispatch}

Economic dispatch (ED) is an approach to minimize the cost of energy production and distribution in a network composed of multiple sources (power plants) by fixing the best load sharing among the sources [52]-[54]. ED is implemented in order to determine the output of each generator so that the total generation cost will be minimized.

The traditional economic dispatch (also called static economic dispatch) method is that of minimizing the production cost to satisfy the load requirements [55]; i.e.

$$
\begin{array}{ll} 
& \min \sum_{i \in I} C_{i}\left(P_{i}(t)\right) \\
\text { s.t. } & \sum_{i \in I} P_{i}(t)=\text { load }+ \text { loss } \\
& P_{i}^{l} \leq P_{i} \leq P_{i}^{u}
\end{array}
$$

where $i$ is the generator index and it is assumed there are a total of $I$ generators in the network. $C$ is the total operating cost over the whole dispatch periods, $T$ is the number of hours in the time horizon, $C_{i}\left(P_{i}(t)\right)$ is the individual generation production cost in terms of real power output $P_{i}$ at time $t$, load is the system load, and loss is the system loss. Usually, smooth quadratic function approximation of cost function is assumed to formulate the ED problem and is expressed as:

$$
C_{i}\left(P_{i}(t)\right)=a_{i} P_{i}^{2}+b_{i} P_{i}+c_{i}
$$

where $a_{i}>0$, and $b_{i}, c_{i} \geq 0$ are constants for the generator ' $\mathrm{i}$ '. 
On the other hand, dynamic economic dispatch (DED) is used to determine dynamically the optimal schedule of online generating output so as to meet the load demand at minimum operating cost under various system and operating constraints over the entire dispatch periods [55]-[56]. It can be think of as the combination of static economic dispatch, optimal power flow (OPF), load frequency control (LFQ), and automatic generation control (AGC). 


\section{CHAPTER III}

\section{MULTI-SOURCE POWER NETWORK WITH FIXED LOADS}

\subsection{System Model}

\subsubsection{Multi-Source Power System Model}

A smart grid delivers electricity from suppliers to consumers, monitors the appliances' consumption and provides consumption guidelines and recommendations to consumers using two-way digital communications. Use of smart grids can save energy, reduce consumption cost and increase reliability of the existing power networks. Consider a smart power network with $N$ loads and $M$ sources (power stations) as shown in Fig. 3.1 for $N=4$ and $M=3$. We assume that every load is equipped with an energy consumption scheduling (ECS) device in its smart meter that announces its consumption schedule at each hour of the day $h \in \mathrm{H}$ where $\mathrm{H}=1,2, \ldots, h_{\max }$ is the scheduling horizon. For example, if the load scheduling is done on a daily basis, $h_{\max }=24$. First, we define maximum source power vector, $\mathbf{p}$, which contains the individual maximum generation capacity of the power stations in the system as: 


$$
\mathbf{p} \triangleq\left[\begin{array}{c}
p_{1} \\
p_{2} \\
\vdots \\
p_{M-1} \\
p_{M}
\end{array}\right]
$$

We also define a load vector, $\mathbf{l}^{h}$, whose entries are the consumption schedules of the loads at hour $h$ that should be sent to the network administrator on an hourly (or daily) basis to help in decision making as:

$$
\mathbf{l}^{h} \triangleq\left[\begin{array}{c}
l_{1}^{h} \\
l_{2}^{h} \\
\vdots \\
l_{N-1}^{h} \\
l_{N}^{h}
\end{array}\right]
$$

We define the load sharing matrix for hour $h, \mathbf{X}^{h}$, as:

$$
\mathbf{X}^{h} \triangleq\left[\begin{array}{ccc}
x_{11}^{h} & \cdots & x_{1 M}^{h} \\
\vdots & \ddots & \vdots \\
x_{N 1}^{h} & \cdots & x_{N M}^{h}
\end{array}\right]
$$

whose entries, $x_{i k}^{h}$, represent the proportion of the total power that must be supplied to load $i$ from source $k$ during hour $h \in \mathrm{H}$. In fact, $x_{i k}^{h}$ shows the percentage of the total generation capacity of source $k, p_{k}$ that is used to feed the load $k$ at hour $h$. These 


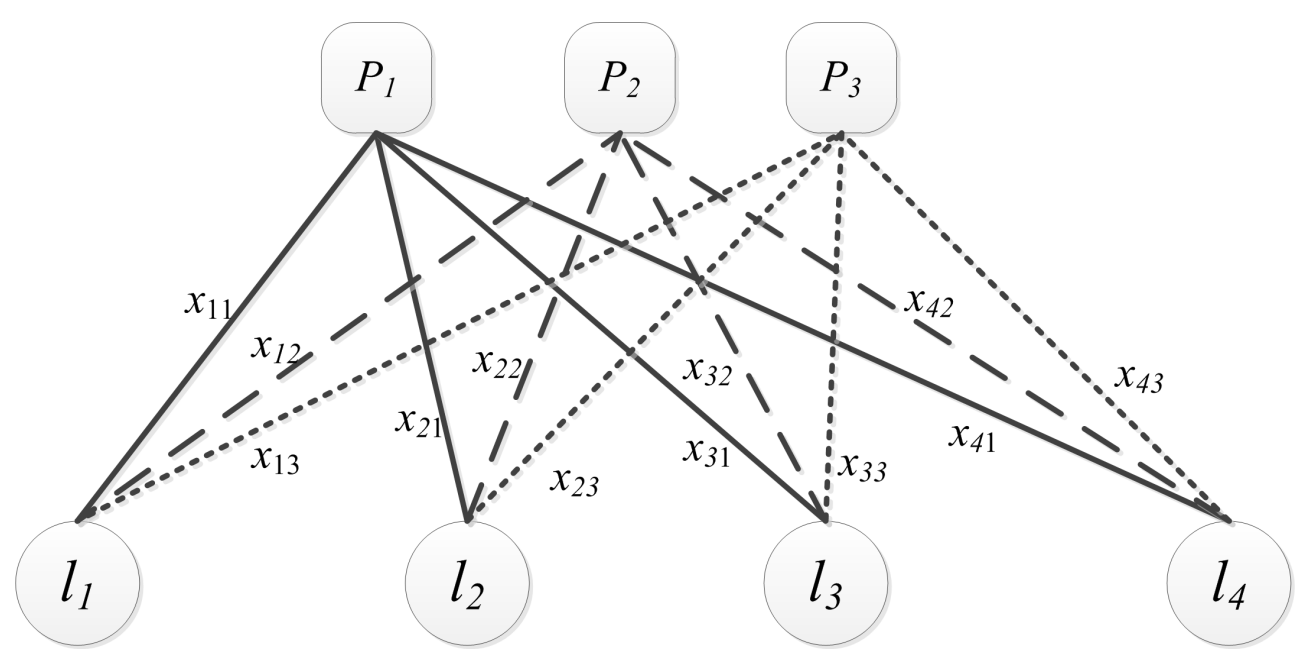

Figure 3.1: A simple interconnected multi-source power network

coefficients are depicted on the link connecting each source unit to loads as shown in Fig. 3.1. To minimize the cost of electricity generation throughout the network, the entries of $\mathbf{X}^{h}$ should be optimized for the entire scheduling horizon. Note that if there is no connectivity between a particular source and load, the corresponding entry in $\mathbf{X}^{h}$ will be preset to zero to show no connectivity. Therefore, we can write the relationship between the load vectors, and the source power vector and the load sharing matrix for every hour, $h \in \mathrm{H}$ as: 


$$
\begin{aligned}
& \mathbf{l}^{h}=\mathbf{X}^{h} \mathbf{p}, \quad \forall h \in \mathrm{H} \\
& \mathbf{l}^{h}=\left[\begin{array}{ccc}
x_{11}^{h} & \cdots & x_{1 M}^{h} \\
\vdots & \ddots & \vdots \\
x_{N 1}^{h} & \cdots & x_{N M}^{h}
\end{array}\right] \times\left[\begin{array}{c}
p_{1} \\
p_{2} \\
\vdots \\
p_{M-1} \\
p_{M}
\end{array}\right]
\end{aligned}
$$

Also, note that for load $i$, the sum of individual powers supplied to it from the $M$ sources should be equal to the load demand by that load at that hour $h \in \mathrm{H}$; that is,

$$
l_{i}^{h}=\sum_{k=1}^{M} x_{i k}^{h} p_{k}, \quad \forall h \in \mathrm{H},(i=1,2, \ldots, N)
$$

where $x_{i k}^{h} p_{k}$ is the hourly fraction of total energy delivered to a load $i$ at each upcoming hour $h \in \mathrm{H}$ from the source $K$. We also define the total energy generated by source $k$ at hour $h$ as $p_{k}^{h}=\sum_{i=1}^{N} x_{i k}^{h} p_{k}$. It is the total energy delivered from source $\mathrm{k}$ to all the loads.

\subsection{Problem Setup and Solution}

In this section, we define three different optimization problems based on the model discussed in Section 3.1 and solve them using numerical optimization techniques. The considered optimization problems are generation cost minimization, PAR minimization and also a hybrid cost function that includes both generation cost and PAR. By 
formulating these problems and solving them, the optimum load sharing coefficients for each hour, i.e. $\mathbf{X}^{\mathbf{h}}$, will be obtained.

\subsubsection{Problem Setup}

\section{Generation Cost Minimization}

Although it is simpler to assume fixed per unit energy generation cost (in terms of cents per $\mathrm{kWh}$ ) for all the sources, here, we consider a variable per unit generation cost for each source. The assumption of variable generation cost has been considered in other papers such as [24] where the generation cost of a source is a quadratic polynomial function of its power output. While our problem formulation is general and independent of the form of the unit generation cost function, in numerical result section we assume a two-step generation cost function for each source as:

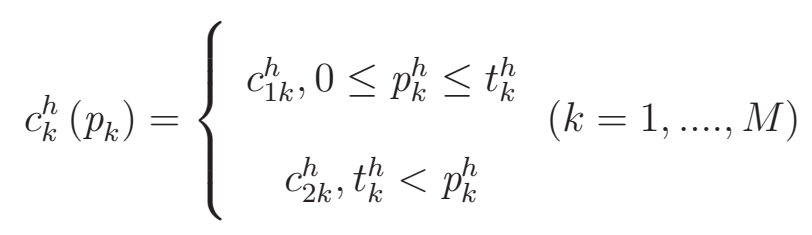

where $t_{k}^{h}$ is the threshold for source $k$ at hour $h, c_{1 k}{ }^{h}$ and $c_{2 k}{ }^{h}$ are the unit generation cost if the required generated energy of source $k$ is below or above the threshold $t_{k}^{h}$, respectively.

Given a feasible energy scheduling set $\mathbf{l}^{h}$, and an RTP scheme given above, the key question is what should be the choice of matrix $\mathbf{X}^{h}$ such that the total cost of energy production is minimized. From the cost function introduced above, the total cost of power production to feed all the $N$ loads from multiple sources within the upcoming 
scheduling horizon is obtained as:

$$
c_{\text {tot }}=c_{k}\left(p_{k}^{h}\right) \times p_{k}^{h}
$$

where the cost function $c_{k}\left(p_{k}^{h}\right)$ is given in (3.6). Having this cost function, we are now ready to formulate the energy distribution problem as the following optimization problem:

$$
\begin{gathered}
\min _{\mathbf{X}^{h}} \sum_{k=1}^{M} c_{k}\left(p_{k}^{h}\right) \times p_{k}^{h} \\
\mathbf{l}^{h}=\mathbf{X}^{h} \mathbf{p} \\
\text { s.t. } \quad 0 \leq \sum_{i=1}^{N} x_{i k}^{h} \leq 1 \\
0<x_{i k}^{h}<1
\end{gathered}
$$

\section{PAR Minimization}

The PAR of energy generation for a source is an important factor in its efficiency, lifetime and long-term maintenance cost. Typical energy sources are more efficient and reliable at lower PARs resulting in lower long-term costs. The PAR of energy generation for a source is defined as the ratio of the maximum energy per hour generated by the source $j$ to the average hourly energy generated by this source throughout the entire scheduling horizon $\mathrm{H}$. Therefore, for the source $j$, we can write the PAR as:

$$
P A R_{j}=\frac{P_{\max }}{P_{\text {avg }}}=h_{\max } \cdot \frac{\max _{h \in \mathrm{H}} \sum_{i=1}^{N} x_{i j}^{h} p_{j}^{h}}{\sum_{h=1}^{H} \sum_{i=1}^{N} x_{i j}^{h} p_{j}^{h}}
$$


To minimize the PAR, the following optimization problem should then be solved in order to find the optimum load sharing coefficients defined in (3.3):

$$
\min _{\mathbf{x}^{h}}\left\{\max _{j}\left(h_{\max } \cdot \frac{\max _{h \in \mathrm{H}} \sum_{i=1}^{N} x_{i j}^{h} p_{j}^{h}}{\sum_{h=1}^{H} \sum_{i=1}^{N} x_{i j}^{h} p_{j}^{h}}\right)\right\} \text { s.t. }\left\{\begin{array}{c}
\mathbf{l}^{h}=\mathbf{X}^{h} \mathbf{p} \\
0 \leqslant \sum_{i=1}^{N} x_{i k}^{h} \leqslant 1 \\
0<x_{i k}^{h}<1
\end{array}\right.
$$

\section{Hybrid Optimization}

Sometimes it is desirable to make a compromise between different measures in a power generation and distribution network. For example, it is possible to combine the generation cost and the PAR measures to set up a hybrid optimization problem to make a trade-off between generation cost and PAR as follows:

$$
\begin{gathered}
\min _{\mathbf{X}^{\mathbf{h}}}\left\{\gamma \sum_{k=1}^{M} c_{k}\left(p_{k}^{h}\right) \times p_{k}^{h}+\max _{j}\left(h_{\max } \cdot \frac{\max _{h \in \mathrm{H}} \sum_{i=1}^{N} x_{i j}^{h} p_{j}^{h}}{\sum_{h=1}^{H} \sum_{i=1}^{N} x_{i j}^{h} p_{j}^{h}}\right)\right\} \\
\text { s.t. } \quad 0 \leq \sum_{i=1}^{N} x_{i k}^{h} \leq 1 \\
0<x_{i k}^{h}<1
\end{gathered}
$$

where $\gamma$ is a weight coefficient. Large $\gamma$ means more emphasis on generation cost and less emphasis on the PAR and vice-versa. In other words, larger $\gamma$ makes the generation cost the dominant metric and the result of the optimization will approach to that of (3.8) and smaller $\gamma$ makes the PAR dominant and the solution will approach to that of (3.9). Note that although the optimization problem in (3.8) minimizes the cost of energy production, it does not necessarily minimize the long-term operation 
cost of the network. The overall cost is the function of both real-time energy generation and long-term maintenance and operation costs. (3.9) can be used to make a balance between these two different types of cost.

\subsubsection{Problem Solution}

In optimization problems of (3.8), (3.10), and (3.11), the connectivity coefficients are all positive. In addition, objective functions and conditions satisfy the convex optimization conditions. Therefore, these three problems are convex optimization problems and can be solved using constrained non-linear optimization or non-linear programming (NLP) techniques such as the interior point method [57]-[60]. In the next section, we use the interior point method to numerically compute the optimum solutions for (3.8), (3.10), and (3.11).

\subsection{Simulation Results}

As a simple example, we consider a network of 3 energy sources and 4 loads. We assume that the cost of energy generation for each source is a two-step function as in (3.6). We assume that the hourly (or daily) consumption schedules of all the loads are known a priori. The loads send their consumption schedules hourly to the grid operator via two-way communication and the operator performs the optimization to find the optimum load sharing coefficients that minimize the overall energy production cost or the PAR throughout the network. 
In the simulation, the required amount of energy per hour for each load has been chosen randomly for a 24-hour span. We then, use non-linear optimization techniques to numerically calculate the optimum load sharing coefficients. First, the cost minimization problems in (3.8) was considered. Based on the known load consumption schedules in a 24-hour span, the optimum coefficients were found using the interior point method [57]-[60]. Fig. 3.2 shows the cost of energy production for a case that uses random arbitrary load sharing coefficients and the same cost when the optimum load sharing coefficients calculated using the proposed cost optimization scheme. The hourly cost for both cases is normalized to show their differences clearly. For comparison, the average cost of energy production was indicated over 24 hours in each case on the plots. As seen, for the first case the average cost of energy production is almost $25 \%$ higher than that of the optimized case. Also, the optimum load sharing coefficients was calculated from the hybrid optimization in (3.11) with $\gamma=1$ and the energy generation cost for this case has also been calculated. As expected, the average cost in this case is slightly higher than that of the cost minimization problem in (3.8) but still a significant cost reduction is obtained compared to the case of random load sharing.

Next, we study the PAR optimization scheme and 15 different random scenarios are generated in which the load consumption schedules are different. Numerical optimization is then performed to find the optimum load sharing for each scenario. In Fig. 3.3, the PAR is plotted as defined in (3.9) for the case of cost optimization in 


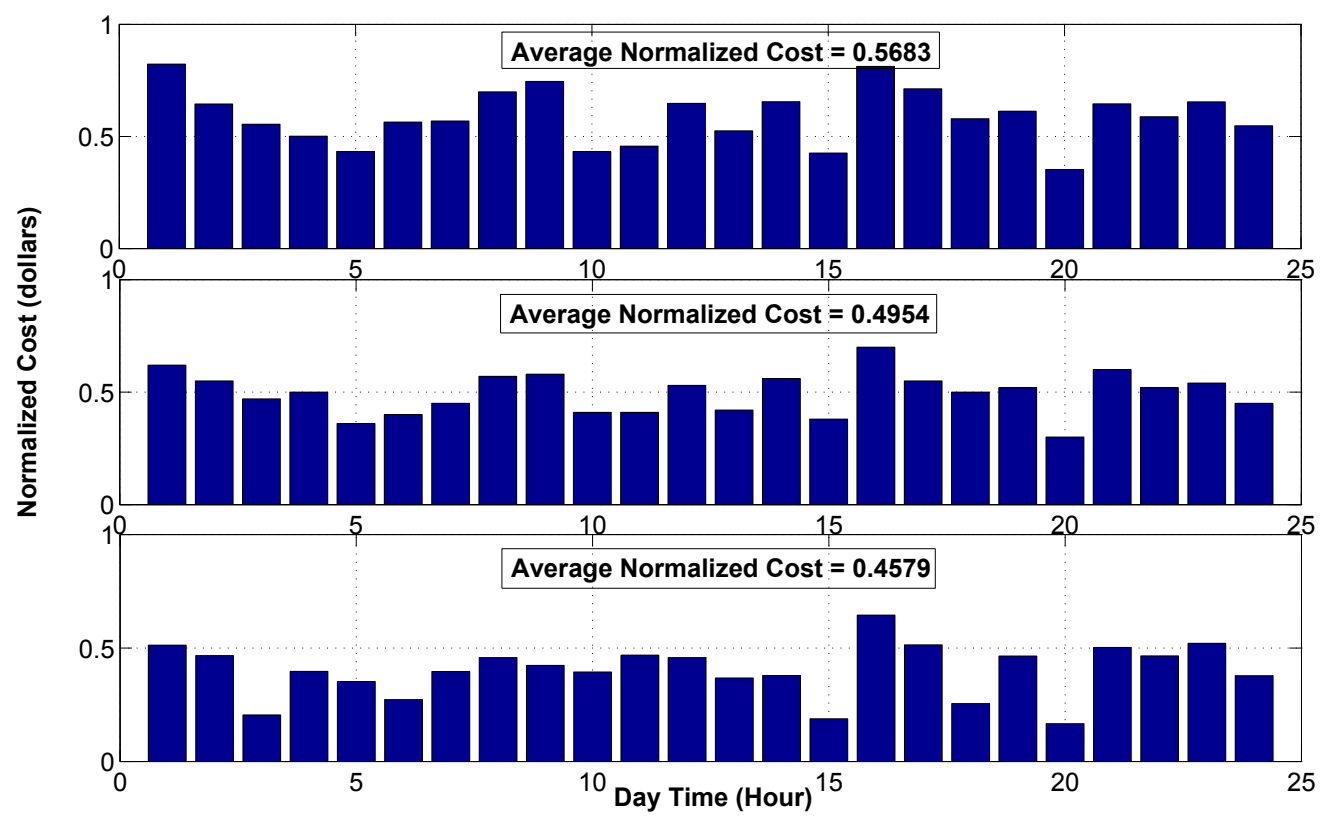

Figure 3.2: Normalized retail total energy cost for $N=4$ users and $M=3$ sources; Top: random load sharing, Center: hybrid load sharing, Bottom: minimum cost load sharing

(3.8) and compared with the case of random load sharing. For reference, the mean of PARs of all the 15 scenarios are also shown. As shown, a $27 \%$ reduction in average PAR is obtained by using the proposed scheme. Therefore, it can be concluded that the proposed cost minimization scheme is also able to reduce the PAR significantly. Next, the problem of PAR minimization in (3.10) is considered. Using the proposed optimization scheme,the optimum load sharing coefficients were find out that minimize the PAR as defined in (3.9) using the interior point method. Fig. 3.4 compares the resulted PAR using this optimization with the PARs obtained from cost optimization and hybrid optimization load sharing schemes for each of the 15 scenarios. As 


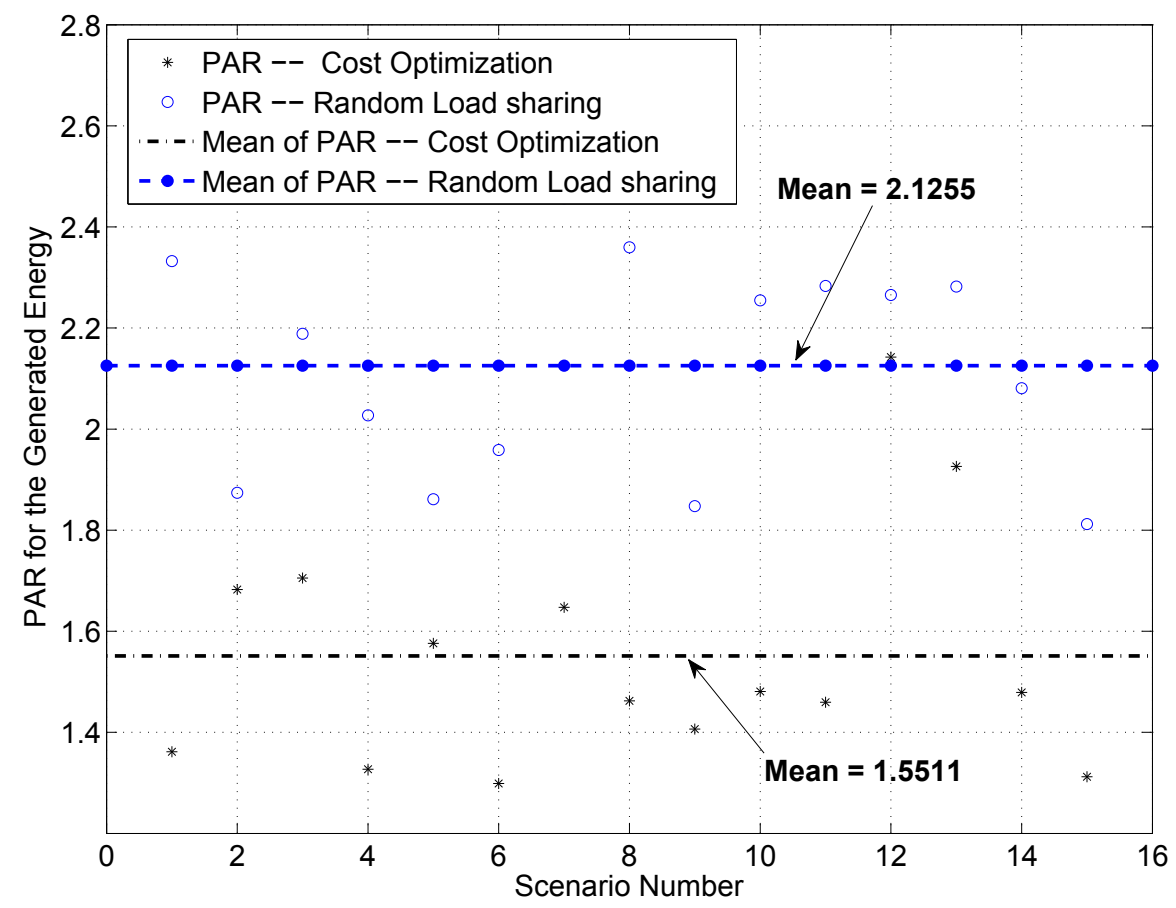

Figure 3.3: PAR for the random and minimum cost load sharing schemes

seen, in all the scenarios the PAR has been reduced. This reduction is also reflected in the mean of PARs averaged over all these 15 scenarios. Using PAR minimization scheme, a reduction of $35 \%, 11 \%$, and $5 \%$ in terms of the average PAR is obtained compared, respectively, to the cases of random, minimum cost and hybrid load sharing schemes.

This chapter of the thesis proposed optimal load sharing schemes to minimize the energy production cost and the PAR in a multi-source power network with fixed or non-shiftable operation loads. The proposed schemes assume that multiple sources 


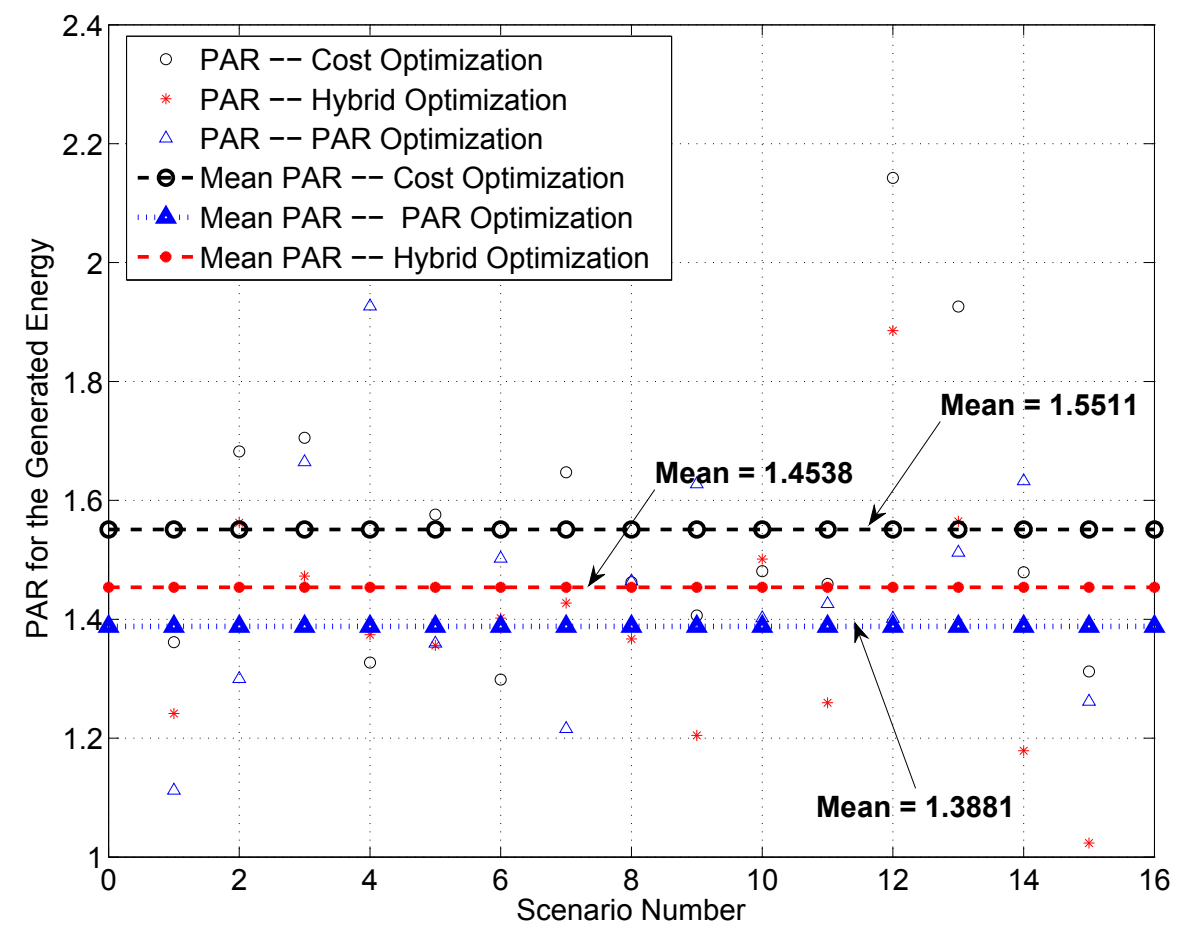

Figure 3.4: PAR for minimum cost, minimum PAR and hyrbid load sharing schemes

connected to multiple loads in the network and that the loads send their consumption schedule to the network administrator on an hourly or a daily basis using two-way communication. The administrator then carries out the optimizations in (3.8), (3.10) and (3.11) to find out the optimum load sharing coefficients. A two-step generation cost function for each source was considered. Simulation results verify that the proposed optimization schemes significantly reduce the cost of energy generation and the PAR in the network. 


\section{CHAPTER IV}

\section{MULTI-SOURCE POWER NETWORK WITH SHIFTABLE LOADS}

\subsection{System Model}

Similar to Chapter 3, we consider a smart power network with $N$ loads and $M$ sources (power stations). However, in this chapter we assume that each load is a shiftable load (a load with flexible consumption schedule vector) and is equipped with an energy consumption scheduling (ECS) device in its smart meter that announces its consumption schedule vector $\mathbf{l}_{n}=\left[l_{n}^{1}, l_{n}^{2}, \ldots ., l_{n}^{H}\right]^{T} \quad n=1,2, \ldots, N$ at each hour of the day $h \in \mathrm{H}$ where $\mathrm{H}=1,2, \ldots, h_{\max }$ is the scheduling horizon.

We also define three vectors $\alpha, \beta$ and $\varepsilon$ to show starting time of consumption, the ending time of consumption, and the electricity usage of a load during the starting and the end times, respectively; i.e.

$$
\begin{aligned}
& \alpha=\left(\alpha_{1}, \alpha_{2}, \ldots, \alpha_{N}\right) \\
& \beta=\left(\beta_{1}, \beta_{2}, \ldots, \beta_{N}\right) \\
& \varepsilon=\left(\varepsilon_{1}, \varepsilon_{2}, \ldots, \varepsilon_{N}\right)
\end{aligned}
$$

where $\alpha_{n}, \beta_{n}$ and $\varepsilon_{n}$ are the starting time, end time, and the load consumption for the $n^{\text {th }}$ load. Maximum source power vector, $\mathbf{p}$, which contains the individual maximum generation capacity of the power stations in the system is defined as follows: 


$$
\mathbf{p} \triangleq\left[\begin{array}{c}
p_{1} \\
p_{2} \\
\vdots \\
p_{M-1} \\
p_{M}
\end{array}\right]
$$

We also define a load vector, $\mathbf{l}^{h}$ at an hour $h$ whose entries are the load requirement of the loads at hour $h$ that should be sent to the network administrator on an hourly (or daily) basis to help in decision making as:

$$
\mathbf{l}^{h} \triangleq\left[\begin{array}{c}
l_{1}^{h} \\
l_{2}^{h} \\
\vdots \\
l_{N-1}^{h} \\
l_{N}^{h}
\end{array}\right]
$$

This load consumption vector relies on the time of load consumption intervals $\alpha$ and $\beta$ as defined in (4.1). For the $n^{\text {th }}$ load, we set all the $l_{n-1}^{h}$ coefficients for which $h$ is not between $\alpha_{n}$ and $\beta_{n}$ to zero. This ensures that no power is allocated to the $n^{\text {th }}$ load outside the time interval of interest. Our optimization scheme then schedules the consumption of the $n^{\text {th }}$ load during to the time interval $\alpha_{n} \leq h \leq \beta_{n}$ as defined 
in 4.1. Finally, we define the load sharing matrix for hour $h, \mathbf{X}^{h}$, as:

$$
\mathbf{X}^{h} \triangleq\left[\begin{array}{ccc}
x_{11}^{h} & \cdots & x_{1 M}^{h} \\
\vdots & \ddots & \vdots \\
x_{N 1}^{h} & \cdots & x_{N M}^{h}
\end{array}\right]
$$

whose entries, $x_{n k}^{h}$, represent the proportion of the total power that must be supplied to load $n$ from source $k$ during hour $h \in \mathrm{H}$. In fact, $x_{n k}^{h}$ shows the percentage of the total generation capacity of source $k$, that is used to feed the load $n$ at hour $h$.

To minimize the cost of electricity generation throughout the network, the entries of $\mathbf{X}^{h}$ should be optimized for the entire scheduling horizon. Note that if there is no connectivity between a particular source and load, the corresponding entry in $\mathbf{X}^{h}$ will be pre-set to zero to show no connectivity. Therefore, we can write the relationship between the load vectors, and the source power vector and the load sharing matrix for every hour, $h \in \mathrm{H}$ as:

$$
\begin{aligned}
\mathbf{l}^{h}= & \mathbf{X}^{h} \mathbf{p}, \\
\mathbf{l}^{h}= & {\left[\begin{array}{ccc}
x_{11}^{h} & \cdots & x_{1 M}^{h} \\
\vdots & \ddots & \vdots \\
x_{N 1}^{h} & \cdots & x_{N M}^{h}
\end{array}\right] \times\left[\begin{array}{c}
p_{1} \\
p_{2} \\
\vdots \\
p_{M-1} \\
p_{M}
\end{array}\right] }
\end{aligned}
$$


Also, note that for load $n$, the sum of individual powers supplied to it from the $M$ sources should be equal to the load demand by that load at that hour $h \in \mathrm{H}$; that is,

$$
l_{n}^{h}=\sum_{k=1}^{M} x_{n k}^{h} p_{k}, \quad \forall h \in \mathrm{H},(n=1,2, \ldots, N)
$$

where $x_{n k}^{h} p_{k}$ is the hourly fraction of total energy delivered to a load $n$ at each upcoming hour $h \in \mathrm{H}$ from the source $k$. We also define the total energy generated by source $k$ at hour $h$ as $p_{k}^{h}=\sum_{n=1}^{N} x_{n k}^{h} p_{k}$. It is the total energy delivered from source $k$ to all the loads. The total daily consumption by each load, i.e. $\varepsilon_{n}$, is defined as

$$
\varepsilon_{n}=\sum_{h=\alpha_{n}}^{\beta_{n}} l_{n}^{h}
$$

and

$$
l_{n}^{h}=0, \forall h \in \mathrm{H} \backslash \mathrm{H}_{\mathrm{n}}
$$

where $\mathrm{H}_{\mathrm{n}}$ is the time interval between $\alpha_{n}$ and $\beta_{n}$. These last two constraints are important as they ensures that each load will be scheduled during its interval of interest.

\subsection{Problem Setup}

In this section, we define two different optimization problems based on the model discussed above and solve them using numerical optimization techniques. Similar to the previous chapter, the considered optimization problems are the energy cost minimization and the PAR minimization. However, an additional degree of freedom 
Table 4.1: System Parameters

\begin{tabular}{|l|l|}
\hline$\varepsilon$ & total daily energy consumption \\
\hline$\alpha$ & starting time of consumption vector \\
\hline$\beta$ & end time of consumption vector \\
\hline $\mathbf{l}^{h}$ & consumption schedule vector at hour $h$ \\
\hline $\mathbf{p}$ & maximum source power vector \\
\hline $\mathbf{X}^{h}$ & load sharing matrix for hour $h$ \\
\hline
\end{tabular}

has been added to the problem due to the flexibility of the loads. Our approach to solve the problem is to use a two-step optimization to come up with an iterative method to find the optimum load schedules. First, assuming a given fixed load schedule we use the optimization methods explained in Chapter 3 to find the best load sharing scheme among the sources for each load and at each hour, i.e. $\mathbf{X}^{h}$. Then, given the calculated load sharing in the previous step as an input, another optimization problem is formulated to find the best load schedules for all the loads $\mathbf{l}^{h}$. These two steps are done in an iterative fashion to find the optimum load sharing and scheduling. Table 4.1 summarizes different system parameters.

\subsubsection{Generation Cost Minimization}

In the first optimization problem, we consider the minimization of the total cost of power generation. Given a feasible flexible energy scheduling set $\mathbf{l}^{h}$, and an IBR 
pricing scheme the key question is what should be the choice of matrix $\mathbf{X}^{h}$ such that the total cost of energy production is minimized. The problem is solved using a two-step optimization.

Step 1: In the first step, assuming a fixed scheduling, the optimum load sharing is obtained. The total cost of power production to feed all the $N$ loads from multiple sources within the upcoming scheduling horizon is obtained as:

$$
c_{\text {tot }}=c_{k}\left(p_{k}^{h}\right) \times p_{k}^{h}
$$

where the cost function $c_{k}\left(p_{k}^{h}\right)$ is the same as in (3.6). Having this cost function, we are now ready to formulate the energy distribution problem as the following optimization problem:

$$
\begin{aligned}
\min _{\mathbf{X}^{h}} & \sum_{k=1}^{M} c_{k}\left(p_{k}^{h}\right) \times p_{k}^{h} \\
& \mathbf{l}^{h}=\mathbf{X}^{h} \mathbf{p} \\
\text { s.t. } & 0 \leq \sum_{i=1}^{N} x_{i k}^{h} \leq 1 \\
& 0<x_{i k}^{h}<1
\end{aligned}
$$

This optimization problem yields an optimized load sharing matrix $\mathbf{X}^{h}$ for hour $h$ that minimizes the cost for energy generation from the source side. The obtained $\mathbf{X}^{h}$ is then used in the next step to update the flexible load schedules with their acceptable scheduling intervals $\alpha$ and $\beta$.

Step 2: In the second step, for a given total (aggregate) power delivered by all the sources at hour $h$, the best load consumption schedule to minimize the cost of energy consumption for all the loads is obtained. This optimization assumes energy 
consumption cost function for the loads. This can be think of as the real rates that the utility company charges the consumers. Although our optimization does not depend on a particular consumption cost function, in the simulation part we will use a quadratic consumption cost function as:

$$
C_{h}\left(l_{n}^{h}\right)=a_{h} l_{n}^{h^{2}}+b_{h} l_{n}^{h}+c_{h}
$$

where $a_{h}>0$ and $b_{h}, c_{h} \geq 0$.

We then formulate an efficient energy consumption scheduling to minimize the energy cost to all the loads which can be expressed as:

$$
\begin{array}{r}
\min _{l_{n}^{h}, \forall n \in N} \sum_{h \in \mathrm{H}} C_{h}\left(\sum_{n \in N} l_{n}^{h}\right) \\
\text { s.t. } \sum_{h \in \mathrm{H}_{\mathrm{n}}} l_{n}^{h}=\varepsilon_{n}
\end{array}
$$

After finding the load consumption schedules from (4.11), Steps 1 and 2 will be repeated in an iterative fashion until a convergence in the cost function is obtained. Table 4.2 summarizes the iterative optimization algorithm to obtain the optimum load sharing coefficients and efficient load consumption schedule.

\subsubsection{PAR Minimization}

In this part, we focus on the optimization of the PAR. The same optimization approach explained above can be equally applied to obtain the optimal load sharing coefficients, $\mathbf{X}^{h}$ to minimize the PAR of the energy sources throughout the scheduling horizon. Like before, the optimization is done in two steps. In the first step, assuming a fixed scheduling, the optimum load sharing is obtained to minimize the 
Table 4.2: Proposed Optimization Algorithm

Algorithm: Finding optimum load sharing coefficients to minimize the cost of energy generation

1: Randomly choose a set of $l_{n}^{h}(n=1,2, \ldots, N)$

\section{2: Repeat}

3: $\quad$ Repeat

4: $\quad$ At each hour Do

5: $\quad$ Solve optimization problem in (4.10)

6: $\quad$ Update $\mathbf{X}^{h}$

7: $\quad$ End

8: $\quad$ Until $\forall h \in \mathrm{H}$

9: $\quad$ Using $\mathbf{X}^{h}$ and load cost scheme on (4.11) Do

10: $\quad$ Solve optimization problem in (4.12)

11: $\quad$ Update $l_{n}^{h}(n=1,2, \ldots, N)$

12: $\quad$ End

13: Until $\mathbf{X}^{h}$ converges to the optimal solution

PAR from the source side. In the second step, assuming the obtained load sharing in the previous step, another optimization problem is formulated to minimize the PAR from the load side. To improve the solution, these two steps are done in an iterative fashion until convergence. 
Step 1: The PAR of the network from the source side at a particular hour $h$ is defined as the ratio of the maximum energy generated amongst all the sources at hour $h$ to the total energy generated by all the sources during hour $h$. Therefore, the PAR for a specific hour $h$ is calculated as:

$$
P A R_{h}=\frac{P \max _{j}^{h}}{P a v g^{h}}=M \cdot \frac{\max _{j} \sum_{i=1}^{N} x_{i j}^{h} p_{j}^{h}}{\sum_{j=1}^{M} \sum_{i=1}^{N} x_{i j}^{h} p_{j}^{h}}
$$

where $i$ is the load index and $j$ is the source index. To minimize the PAR, the following optimization problem should then be solved in order to find the optimum load sharing coefficients defined in (4.4):

$$
\min _{\mathbf{x}^{h}}\left\{M \cdot \frac{\max _{j} \sum_{i=1}^{N} x_{i j}^{h} p_{j}^{h}}{\sum_{j=1}^{M} \sum_{i=1}^{N} x_{i j}^{h} p_{j}^{h}}\right\} \text { s.t. }\left\{\begin{array}{c}
\mathbf{l}^{h}=\mathbf{X}^{h} \mathbf{p} \\
0 \leq \sum_{i=1}^{N} x_{i k}^{h} \leq 1 \\
0 \leq x_{i k}^{h} \leq 1
\end{array}\right.
$$

Step 2: Considering the the optimal load sharing coefficients obtained from the optimization in the first step, we can formulate a PAR minimization scheme at the load side to find out the best load scheduling. The PAR for the load distribution can be calculated as follows:

$$
P A R=\frac{L_{\text {peak }}}{L_{\text {avg }}}=h_{\text {max }} \cdot \frac{\max _{h \in \mathrm{H}} \sum_{n \in N} l_{n}^{h}}{\sum_{n \in N} \varepsilon_{n}}
$$

Therefore, given the PAR equation in (4.15), the following optimization problem can be formulated to find the best load consumption schedule to minimize the PAR: 


$$
\begin{array}{r}
\min _{l_{n}^{h}, \forall n \in N}\left\{h_{\text {max }} \cdot \frac{\max _{h \in \mathrm{H}} \sum_{n \in N} l_{n}^{h}}{\sum_{n \in N} \varepsilon_{n}}\right\} \\
\text { s.t. } \sum_{h \in \mathrm{H}_{\mathrm{n}}} l_{n}^{h}=\varepsilon_{n}
\end{array}
$$

Next, note that since $h_{\max }$ and $\sum_{n \in N} \varepsilon_{n}$ are fixed as far as the optimization variables are concerned, they can be removed from the objective function and we can rewrite the optimization problem in (4.16) as the following equivalent problem:

$$
\begin{array}{r}
\min _{l_{n}^{h}, \forall n \in N}\left\{\max _{h \in \mathrm{H}} \sum_{n \in N} l_{n}^{h}\right\} \\
\text { s.t. } \sum_{h \in \mathrm{H}_{\mathrm{n}}} l_{n}^{h}=\varepsilon_{n}
\end{array}
$$

The above two steps can be done in an iterative fashion to further improve the load sharing coefficients and the load consumption schedules.

\subsection{Simulation Results}

This section provides the simulation results to assess the performance of the proposed optimization algorithms. Throughout the simulations, we consider $N=4$ and $M=3$. All the loads are assumed to be flexible or shiftable within a time interval. The loads are randomly generated such that the total load connected to the network does not exceed the total generation capacity at each hour.

First, we consider the cost minimization problem. The energy consumption cost function is assumed to be quadratic as in (4.11). For simplicity, it is assumed that 
$b_{h}=c_{h}=0$ for all $h \in \mathrm{H}$. Also, it is assumed that $a_{h}=0.3$ at day-time hours, i.e. from 8:00AM to 12:00PM, and $a_{h}=0.2$ during the night hours, i.e. from 12:00PM to 8:00AM. The iterative optimization introduced in the previous section is used to find out the optimum load sharing coefficients. It turns out that after about five iterations the optimization algorithm on verges to the optimal solutions.

Fig. 4.1 and Fig. 4.2 show the load schedules before and after optimization for a single scenario. As seen, the load schedules have changed from the initial schedule to a different schedule to minimize the total cost of energy generation. Notice that the scheduling interval for all the four loads, i.e. $\left[\alpha_{n}, \beta_{n}\right] n=1,2,3,4$ have remained the same in both figures; however, the load scheduling patterns have changed from Fig. 4.1 to Fig. 4.2. Fig. 4.3 shows the daily cost of electricity per load before and after optimization. As seen, for all the loads, the cost has decreased after optimization. By computing the total cost of energy generation and the PAR of the system, we find out that the total cost has reduced from $\$ 23.04$ to $\$ 18.99$ (i.e. $17.56 \%$ cost reduction) and the PAR from 7.1027 to 5.808 (i.e. $18.23 \%$ reduction) which further proves the efficiency of the proposed optimization scheme.

\subsubsection{PAR Minimization}

To demonstrate the efficiency of the PAR minimization scheme, we consider 22 different simulation scenarios flexible load and perform our optimization schemes for each case. In Fig. 4.4, the PAR with optimization scheme, the PAR achieved through the cost optimization scheme and the PAR achieved through the PAR optimization 

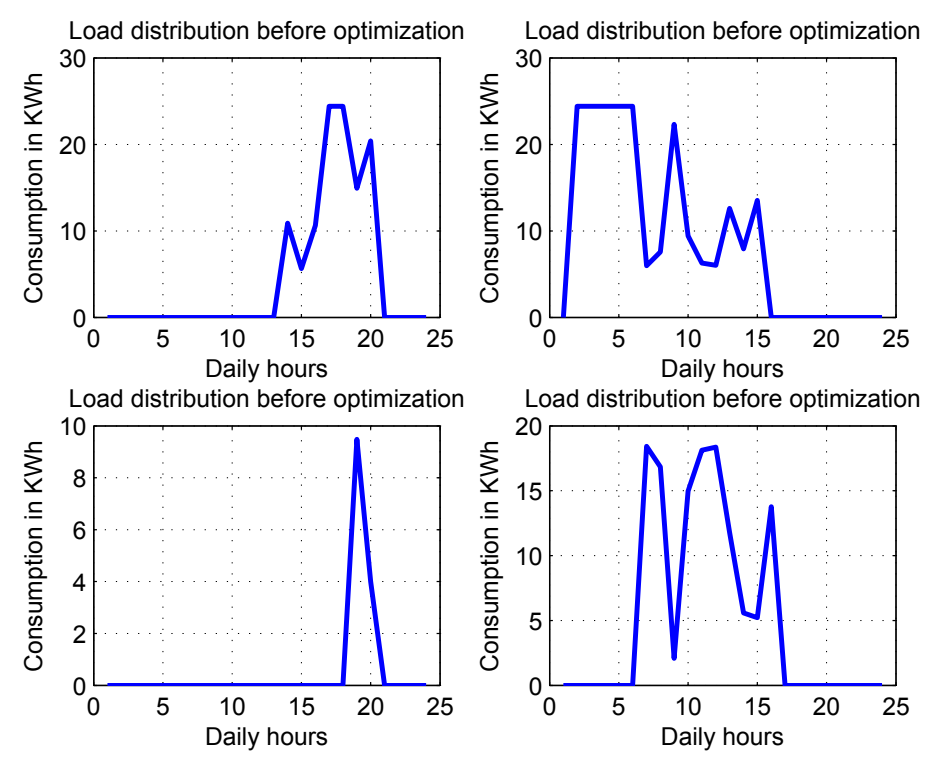

Figure 4.1: Load distribution before optimization

scheme have been plotted. After running the simulation for 22 different scenarios, it turns out that in almost all the scenarios a reduction in the PAR is obtained. However, there are certain scenarios that the PAR reduction is negligible. We have also plotted the average value of the PARs for 22 scenarios. As seen the average PAR due to applying the proposed PAR minimization scheme is significantly lower than the other two cases. This further proves that the PAR minimization scheme works efficiently. It is also worth mentioning that the resulted PAR is lower when the allowed scheduling intervals for the loads are widen. This allows for better distribution of loads and may result in a lower PAR. It has been shown in [29] that the presence of shiftable loads in the network can help in reducing the PAR. 

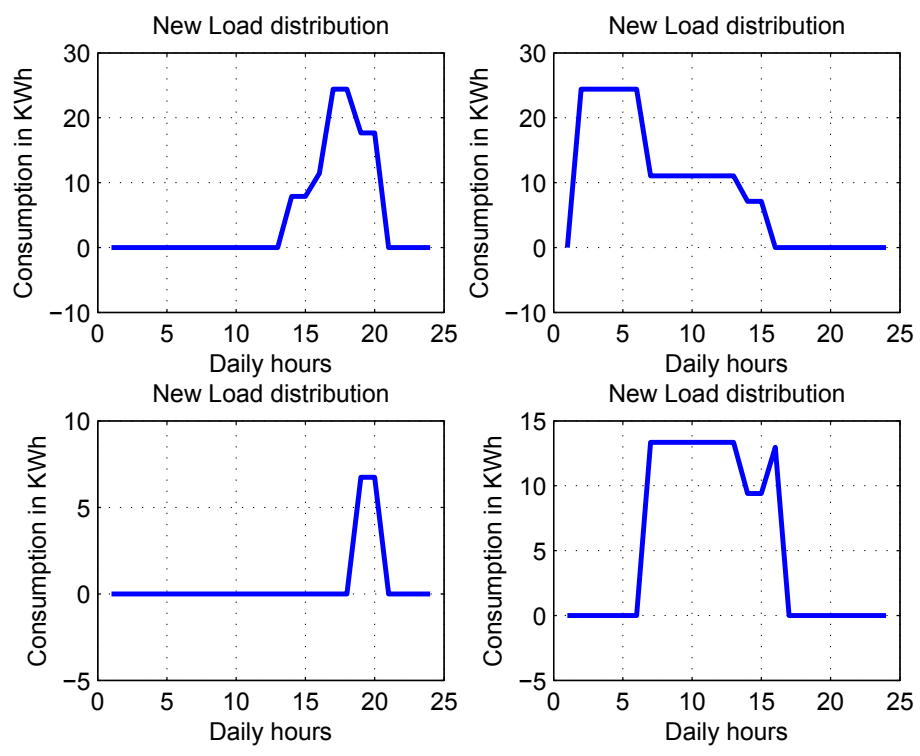

Figure 4.2: Load distribution after optimization over the interval of consumption

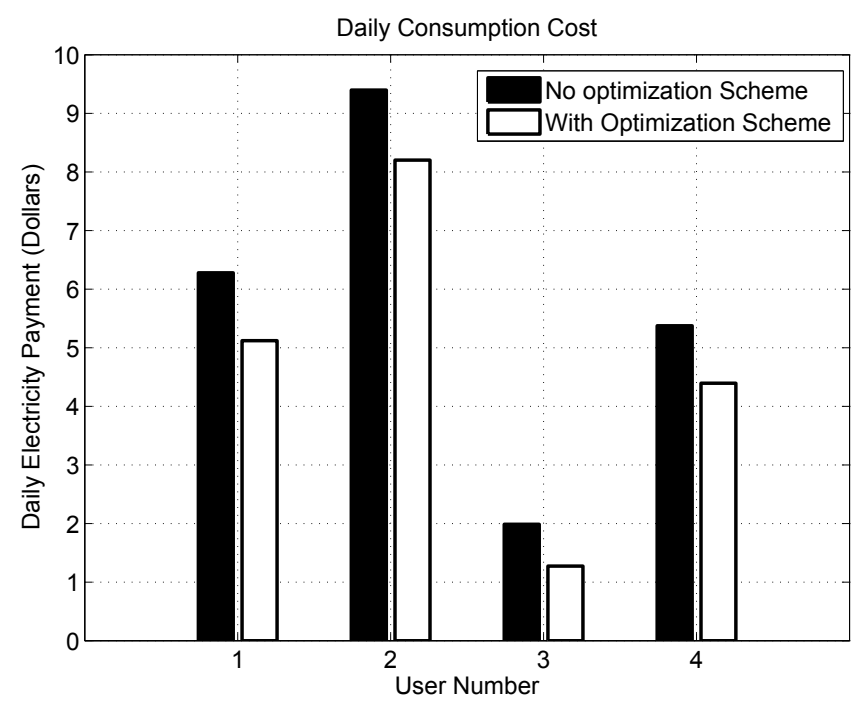

Figure 4.3: Daily charges for each load before and after optimization 


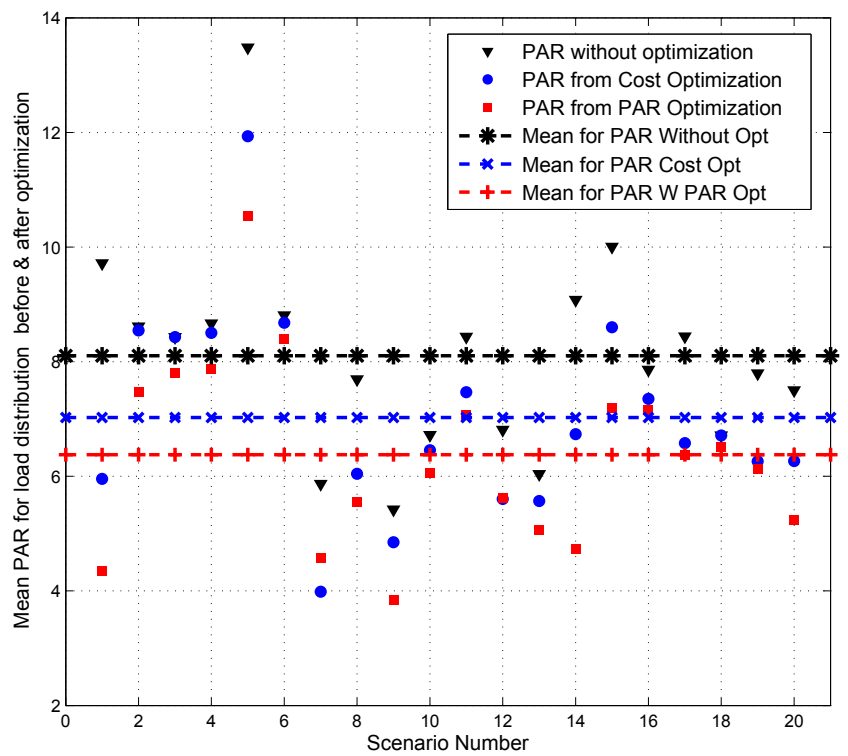

Figure 4.4: PAR performance: without optimization, with cost minimization, and with PAR minimization schemes 


\section{CHAPTER V}

\section{CONCLUSION}

The current power generation and distribution networks are not optimized. It is required to make them more efficient to cope with the constant increase in energy demand. Regardless of how quickly various utilities will accept smart grid concept, they all agree on the necessity of the optimization of their power system performance. This thesis considered the optimization of a multi-source multi-load power network that employs two-way communication among sources and loads. We considered the optimization of several system performance metrics such as the energy generation cost and the peak-to-average ratio (PAR) of power generation at each source.

Several optimization schemes were defined for both shiftable and non-shiftable loads that are connected to the multi-source network and optimization algorithms were developed to optimize the cost and the PAR based on the type of loads in the system. We also considered a hybrid optimization problem in which the optimization target function is a weighted sum of the cost and the PAR metrics.

Simulation results show an improved energy cost and PAR performance for both shiftable and non-shiftable load cases. For non-shiftable loads where the loads are distributed over the entire scheduling horizon, a reduction of $35 \%$ and $20 \%$ in the energy cost and the PAR performance was obtained. While considering shiftable 
loads that are restricted to operate on a given time interval of operation, it was possible to get a reduction of $18 \%$ for both the cost and the PAR. Generally, it was shown that the existence of shiftable loads in a power network can enhance the performance of the system in terms of energy generation cost and the PAR. 


\section{BIBLIOGRAPHY}

[1] A. Sripetch and P. Saengudomlert, "Topology Design of Optical Networks Based on Existing Power Grids," Fifth Annual Conference on Communication Networks and Services Research, 200\%. CNSR 'O7, pp. 35-40, 14-17 May 2007.

[2] J. McDonald, "Leader or follower: developing the smart grid business case," IEEE Power and Energy Magazine, vol. 6, no. 6, pp. 18-90, Nov. 2008.

[3] P. K. Steimer, "Enabled by high power electronics - Energy efficiency, renewables and smart grids," 2010 International Power Electronics Conference (IPEC), pp. 11-15, 21-24 June 2010.

[4] R. Podmore, R. Larsen, H. Louie, and B. Waldron, "Affordable energy solutions for developing communities," 2011 IEEE Power and Energy Society General Meeting, pp. 1-8, 24-29 July 2011.

[5] European technology platform smart grids, 2011 Report, available at: http ://www.smartgrids.eu 2010.

[6] P. Wolfs and S. Islam, "Potential barriers to smart grid technology in Australia," Curtin University of Tech., Perth, Australia, 2008.

[7] O. Gulich, "Technological and business challenges of Smart Grids," Lappeenranta University of Tech., 2009.

[8] A. Moshari, G. R. Yousefi, A. Ebrahimi, and S. Haghbin, "Demand-side behavior in the smart grid environment," 2010 IEEE PES Innovative Smart Grid Technologies Conference Europe, pp. 1-7, 11-13 Oct. 2010.

[9] "Materials from the smart grid workshop in Fortum, Oy," Jan. 142010.

[10] D. Leeds, "The smart Grid in 2010," GTM Research, July 2010.

[11] Y. Julliard, "How to smarten up your Grid," E21 conf., Melbourne, July 2009. 
[12] R. D. Tabors, G.Parker, and M. C. Caramanis, "Development of the Smart Grid: Missing Elements in the Policy Process," 43rd Hawaii International Conference on System Sciences (HICSS), 2010, pp. 1-7, 5-8 Jan. 2010.

[13] R. E. Schuler, "Electricity Markets, Reliability and the Environment: SmarteningUp the Grid," 43rd Hawaii International Conference on System Sciences (HICSS), 2010, pp. 1-7, 5-8 Jan. 2010.

[14] B. Ramanathan and V. Vittal, "A framework for evaluation of advanced direct load control with minimum disruption," IEEE Trans. on Power Systems, vol. 23, no. 4, pp. 1681-1688, Nov. 2008.

[15] C. W. Gellings and J. H. Chamberlin, "Demand Side Management: Concepts and Methods," 2nd ed., PennWell Books, 1993.

[16] M. A. A. Pedrasa, T. D. Spooner, and I. F. MacGill, "Scheduling of demand side resources using binary particle swarm optimization," IEEE Trans. Power Systems, vol. 24, no. 3, pp. 1173-1181, Aug. 2009.

[17] M. S. Majid, H. A. Rahman, M. Y. Hassan, and C. A. Ooi, "Demand side management using direct load control for residential," SCOReD 2006, pp. 241245, June 27-28, 2006.

[18] H. Lee and C. L. Wilkins, "A practical approach to appliance load control analysis: A water heater case study," IEEE Trans. Power App. Syst.,vol. PAS102, pp. 1007-1013, Apr. 1983.

[19] A. Cohen and C. Wang, "An optimization method for load management scheduling," IEEE Trans. Power Syst., vol. 3, no. 2, pp. 612-618, May 1988.

[20] C. N. Kurucz, D. Brandt, and S. Sim, "A linear programming model for reducing system peak through customer load control programs," IEEE Trans. Power Syst., vol. 11, no. 4, pp. 1817-1824, Nov. 1996.

[21] Z. Popovic, "Determination of optimal direct load control strategy using linear programming," CIRED 1999, Nice, France, 1999.

[22] W. Chu, B. Chen, and C. Fu, "Scheduling of direct load control to minimize load reduction for a utility suffering from generation shortage," IEEE Trans. Power Syst., vol. 8, no. 4, pp. 1525-1530, Nov. 1993.

[23] K. Ng and G. Sheble, "Direct load control - A profit based load management 
using linear programming," IEEE Trans. Power Syst., vol. 13, no. 2, pp. 688694, May 1998.

[24] A. K. Zadeh, K. M. Nor, and H. Zeynal, "Multi-thread security constraint economic dispatch with exact loss formulation," 2010 IEEE International Conference on Power and Energy (PECon), pp. 864-869, Nov. 29 - Dec. 12010.

[25] N. Ruiz, I. Cobelo, and J. Oyarzabal, "A direct load control model for virtual power plant management," IEEE Trans. Power Systems, vol. 24, no. 2, pp. 959-966, May 2009.

[26] S. Panta, S. Premrudeepreechacharn, S. Nuchprayoon, C. Dechthummarong, S. Janjommanit, and S. Yachiangkain, "Optimal economic dispatch for power generation using artificial neural network," 2007 International Power Engineering Conference, IPEC 200\%, pp. 1343-1348, Dec. 3-6, 2007.

[27] R. Bharathi, M. J. Kumar, D. Sunitha, and S. Premalatha, "Optimization of combined economic and emission dispatch problem: A comparative study," 2007 International Power Engineering Conference, IPEC 200\%, pp. 134-139, Dec. 3-6, 2007.

[28] A. K. Zadeh, K. M. Nor, and H. Zeynal, "Multi-thread security constraint economic dispatch with exact loss formulation," 2010 IEEE International Conference on Power and Energy (PECon), pp. 864-869, Nov. 29 - Dec. 12010.

[29] A. Mohsenian-Rad, V. W. S. Wong, J. Jatskevich, and R. Schober, "Autonomous demand side management based on game-theoretic energy consumption scheduling for the future smart grid," IEEE Trans. Smart Grid, vol. 1, no. 3, pp. 320-331, Aug. 2010.

[30] A. H. Mohsenian-Rad and A. Leon-Garcia, "Optimal residential load control with price prediction in real-time electricity pricing environments," IEEE Trans. Smart Grid, vol. 1, no. 2, pp. 120-133, Sep. 2010.

[31] A. J. Wood and B. F. Wollenberg, Power Generation, Operation, and Control, New York, NY: John Wiley and Sons, 1996.

[32] S. Panta, S. Premrudeepreechacharn, S. Nuchprayoon, C. Dechthummarong, S. Janjommanit, and S. Yachiangkain, "Optimal economic dispatch for power generation using artificial neural network," International Power Engineering Conf. 200\%, IPEC 200\%, pp. 1343-1348, 3-6 Dec. 2007. 
[33] R. Bharathi, M. J. Kumar, D. Sunitha, and S. Premalatha, "Optimization of combined economic and emission dispatch problem: A comparative study," International Power Engineering Conf. 2007. IPEC 2007, pp. 134-139, 3-6 Dec. 2007.

[34] A. K. Zadeh, A.K.; Nor, K.M.; Zeynal, H.; , "Multi-thread security constraint economic dispatch with exact loss formulation," IEEE International Conference on Power and Energy (PECon), vol., no., pp. 864-869, Nov. 29 2010-Dec. 12010.

[35] "DSM best practices guidebook," IIEC, July 2006.

[36] G. T. Bellarmine, "Load management techniques," IEEE Southeast Conf. 2000, pp. 139-145, 2000.

[37] "DSM and Demand Response in Municipalities," IndEco, January, 2004.

[38] L. Goldberg, "Smart Energy 2.0 Profile", available at: http ://www.digikey.com/ zigbees-smart-energy-20-profile.html Dec. 2009.

[39] A. Faruqui and J. Palmer, "Dynamic Pricing and its discontents," The Battle Group, Aug. 2011.

[40] W. Zhang and A. Feliachi, "Residential load control through real-time pricing signals," Proceedings of the 35th Southeastern Symposium on System Theory, pp. 269- 272, 16-18 Mar. 2003.

[41] P. Reiss and M. White, "Household electricity demand revisited," Rev. Econ. Studies, vol. 72, no. 3, pp. 853-883, July 2005.

[42] A. H. Mohsenian-Rad, V. Wong, J. Jatskevich, and R. Schober, "Optimal and autonomous incentive based energy consumption scheduling algorithm for Smart Grid," IEEE PES Conf. Innov. Smart Grid Technol., Gaithersburg,MD, Jan. 2010 .

[43] P. Samadi, A. Mohsenian-Rad, R. Schober, V. W. S. Wong, and J. Jatskevich, "Optimal Real-Time Pricing Algorithm Based on Utility Maximization for Smart Grid," 2010 First IEEE International Conference on Smart Grid Communications (SmartGridComm), pp.415-420, 4-6 Oct. 2010.

[44] T. Flaim, "Dynamic Pricing in a Smart Grid world," Energy Resources economics, LLC, Jan. 2010. 
[45] S. Datchanamoorthy, S. Kumar, Y. Ozturk, and G. Lee, "Optimal time-of-use pricing for residential load control," 2011 IEEE International Conference on Smart Grid Communications (SmartGridComm), pp.375-380, 17-20 Oct. 2011.

[46] P. Malisani, B. Favre, S. Thiers, B. Peuportier, F. Chaplais, and N. Petit, "Investigating the ability of various buildings in handling load shiftings," 2011 IEEE Power Engineering and Automation Conference (PEAM), vol. 2, pp. 393-397, 8-9 Sept. 2011.

[47] G. M. Masters, "Renewable and effecient electric power systems," John Wiley Publication, New Jersey, 2004.

[48] J. Zhang, X. Xia and D. Alexander "Demand side optimal strategy for Voluntary Load shedding," IEEE Conf. on Power Systems, Nov. 2009.

[49] W. Choudhury and S. Andrews, "Payment mechanisms for micro-generation," DTI report, 2002.

[50] M. Newborough and P. Augood, "Demand side Management opportunities for the UK domestic sector," IEEE Proceedings Generation, Transmission and Distribution, vol. 146, no. 3, pp. 283-293, May. 1999.

[51] I. Cobelo, "active control of distribution networks," University of Manchester, 2005 .

[52] L. K. Kirchamayer, "Economic Operation of Power Systems," Wiley Production, New York, 1988.

[53] J. Z. Zhu and M. R. Irving, "Combined active and reactive dispatch with multiple objectives using analytic hiararchical process," IEE Proc. C, vol. 143, no. 4, pp. 344-352, 1996.

[54] M. R. Irving and M. J. H. Sterling, "Economic Dispatch of Active Power With constraint Relaxation ," IEE Proc. C, vol. 130, no. 4, 1983.

[55] A. M. A. A. Joned, I. Musirin, and T. K. Abdul Rahman, "Solving Dynamic Economic Dispatch Using Evolutionary Programming," 2006 IEEE International Power and Energy Conference, PECon '06 , pp. 144-149, 28-29 Nov. 2006 .

[56] J. Zhu, X. Xiong, S. Lou, M. Liu, Z. Yin, B. Sun, and C. Lin, "Two stage approach for economic power dispatch," 2008 IEEE Power and Energy Society 
General Meeting - Conversion and Delivery of Electrical Energy in the 21st Century, pp. 1-5, 20-24 July 2008.

[57] S. Boyd and L. Vandenberghe, Convex Optimization, Cambridge, UK: Cambridge University Press, 2004.

[58] D. P. Bertsekas, Nonlinear Programming, 2nd ed., Athena Scientific, 1999.

[59] A. Forsgren, P. E. Gill, and M. H. Wright, "Interior methods for nonlinear optimization," SIAM Review, vol. 44, no. 4, pp. 525-597, 2002.

[60] S. Mehrotra, "On the implementation of a primal-dual interior point method," SIAM J. Optimization, vol. 2, no. 4, pp. 575-601, 1992. 